\title{
Quantum Landauer erasure with a molecular nanomagnet
}

\author{
R. Gaudenzi ${ }^{1}{ }^{*}$ E. Burzurí ${ }^{1}$, S. Maegawa ${ }^{2}$, H. S. J. van der Zant ${ }^{1}$, and F. Luis ${ }^{3}$ \\ ${ }^{1}$ Kavli Institute of Nanoscience, Delft University of Technology, 2600 GA, Delft, The Netherlands \\ ${ }^{2}$ Graduate School of Human and Environmental Studies, Kyoto University, Kyoto 606-8501, Japan and \\ ${ }^{3}$ Instituto de Ciencia de Materiales de Aragón (ICMA), \\ C.S.I.C.-Universidad de Zaragoza, E-50009 Zaragoza, Spain
}

The erasure of a bit of information encoded in a physical system is an irreversible operation resulting in a minimal entropy production $k_{\mathrm{B}} \ln 2 .^{1}$ While this limit, called Landauer limit, has been demonstrated to hold for a variety of classical systems, there is no definite proof that it can be extended to the quantum realm, where quantum superposition of discrete energy eigenstates take the place of thermal fluctuations in a continuous spectrum. Here, we use a crystal of molecular nanomagnets as a spin-memory device and show that the Landauer limit is also applicable to quantum systems. In contrast to the other classical systems, the limit is bordered while preserving fast operation thanks to the tunable and fast quantum dynamics. This result explores the thermodynamics of quantum information and suggests a way to enhance classical computations by using quantum processes.

While a computation performed with an ideal binary logic gate (e.g. NOT) has no lower energy dissipation limit $^{5,6}$, one carried out in a memory device does. The reason is that in the former the bit is merely displaced isentropically in the space of states, whereas in the latter the minimal operation, called Landauer erasure, entails resetting the memory irrespective of its initial state. Let us see how this erasure applies to a classical $N$-bit register (Fig. 1(a, left)) and how the Landauer limit comes about. In the first stage, each bit of the register, in a definite state ' 0 ' or ' 1 ', is let to explore the two binary states by lowering the potential barrier and through the action of temperature fluctuations. This doubling of the phase space is accompanied by an entropy production $\Delta S=k_{\mathrm{B}} \ln 2$ per bit. In the second stage, a work $W \geq T \Delta S$ is required to reduce the register's entropy and phase space to their initial values. The limit $W=T \Delta S$ is reached only if this reduction is carried out reversibly. This can be achieved when using a frictionless system in a quasi-static fashion, i.e., at timescales slower than its relaxation time $\tau_{\text {rel }}$, so that unwanted memory and hysteresis effects are avoided. For this reason, slow (fast) operation - with respect to the system-dependent $\tau_{\text {rel }}-$ is generally associated with a lower (higher) dissipation.

This complementarity between work and time suggests considering the product $W \cdot \tau_{\text {rel }}$ - rather than either of the two - as the figure of merit assessing the energy-time cost of a computation. On one hand, driven by the demand for speed, effort has been put in pursuing fast-switching

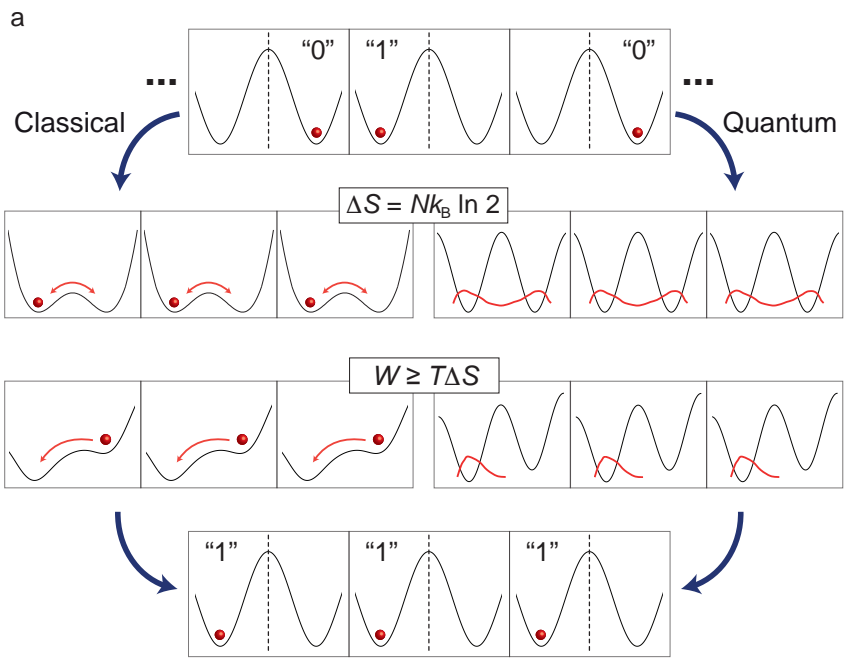

b
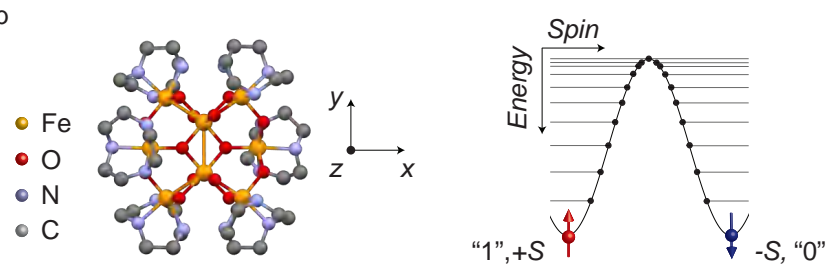

FIG. 1. Quantum-enhanced Landauer erasure and storage of a molecular bit. (a) Schematics of the Landauer erasure process. In order to erase a classical bit register, the effective barriers separating the two binary states of each bit are lowered and thermal fluctuations allow the system to fluctuate between these quasi-degenerate states. A small bias is then applied to increase, within a time $\tau_{\text {rel }}$, the equilibrium population in the desired potential well and store the new information state (here '1'). The Landauer principle fixes the minimal entropy production $\Delta S$ and work $W$ involved in the erasure process. In quantum mechanics, the process changes the wave functions describing the bit states (only the ground state is shown for clarity). (b) Sketch of the $\mathrm{Fe}_{8}$ molecular magnet. In the absence of a magnetic field, the double-well potential favors the two $S_{z}= \pm 10$ spin eigenstates.

storage devices. This has successfully produced state-ofthe-art systems with picosecond timescales, though operating far $\left(\gtrsim 10^{6}\right)$ above the reversible limit ${ }^{7-10}$. On the other hand, reducing $W$ down to the Landauer limit, at the expense of slow operation, has been beautifully demonstrated using small particles in traps ${ }^{13,14}$ or singledomain nanomagnets ${ }^{15}$ as envisioned by Landauer and Bennett. ${ }^{1,2}$ 
All the mentioned systems are large enough to be treated as classical. When the bit is realized with a quantum system, the Landauer erasure protocol leads to changes, namely de- and re-localization, in the wavefunctions describing the bit states, as Fig. 1 (a, right) illustrates. One might then wonder if the Landauer limit remains still applicable to such situations, and if phenomena like quantum tunneling can help reducing the energy-time cost of a computation. Such questions concern the thermodynamics of quantum systems and are $a$ priori non-trivial. ${ }^{11,12}$ Here, we provide a experimental framework that allows to explore them.

In our experiment, a crystal of $\mathrm{Fe}_{8}$ molecular magnets $(\mathrm{MMs})^{17}$ is used as a quantum spin memory to perform the Landauer erasure. The molecular size and net spin lie in between those of magnetic nanoparticles and of paramagnetic ions. At low temperatures, these molecular clusters show magnetic hysteresis, thus magnetic memory, ${ }^{17,18}$ and display genuinely quantum phenomena, such as spin tunneling, ${ }^{19}$ quantum spin interference $^{18,20}$ and quantum phase transitions. ${ }^{16}$

Each $\mathrm{Fe}_{8}$ molecule represents a magnetic bit and is composed of eight spin- $\frac{5}{2} \mathrm{Fe}^{3+}$-ions coupled to each other by competing antiferromagnetic interactions to form a collective $S=10\left(20 \mu_{\mathrm{B}}\right)$ giant-spin. By bottom-up chemical synthesis, arrays of these MMs, with perfectly aligned magnetic axes, are packed into a single crystal. Due to the relatively large intermolecular spacing, the exchange interactions between the molecules are negligible. ${ }^{16}$ The giant-spin $S=10$ multiplet of a single MM is described by the following Hamiltonian ${ }^{17}$ :

$$
\mathcal{H}=-D S_{z}^{2}+E\left(S_{x}^{2}-S_{y}^{2}\right)-g \mu_{B} \boldsymbol{S} \cdot \boldsymbol{B} .
$$

The ligand field, parameterized by the anisotropy constants $D=0.294 \mathrm{~K}$ and $E=0.046 \mathrm{~K}$, defines $x, y$ and $z$ as the hard, medium and easy magnetic axes, respectively, and creates an effective energy barrier separating the $S_{z}= \pm 10$ ground eigenstates that encode the ' 0 ' and '1' bit states (Fig. 1(b), right). The third (Zeeman) term of Eq. (1) accounts for the interaction with a magnetic field, which gives the external control over the potential energy landscape that is required to carry out the Landauer erasure. The effects of different magnetic field components are depicted in Fig. 2(a), which shows the classical potential and the quantum energy levels calculated from Eq. (1). A magnetic field $H_{y}$, applied along the medium axis, allows to tune the height of the potential energy barrier without breaking the degeneracy between ' 0 ' and ' 1 '. In a quantum system, this transverse magnetic field promotes the quantum mixing of "up" and "down" spin orientations. Spins are then able to tunnel through the barrier via progressively lower lying levels, thus leading to an effectively lower activation energy $U$ for the spin reversal and a consequently shorter spin relaxation time $\tau_{\text {rel }} .{ }^{21}$ For $\mathrm{Fe}_{8}, \tau_{\text {rel }}$ approximately follows Arrhenius' law $\tau_{\text {rel }}=\tau_{0} \exp U / k_{\mathrm{B}} T$, where $U$ is determined by the dominant tunneling channel and $\tau_{0} \simeq 10^{-8}$ $\mathrm{s}$ is an attempt time (see Fig. 2(a) and Appendix C).

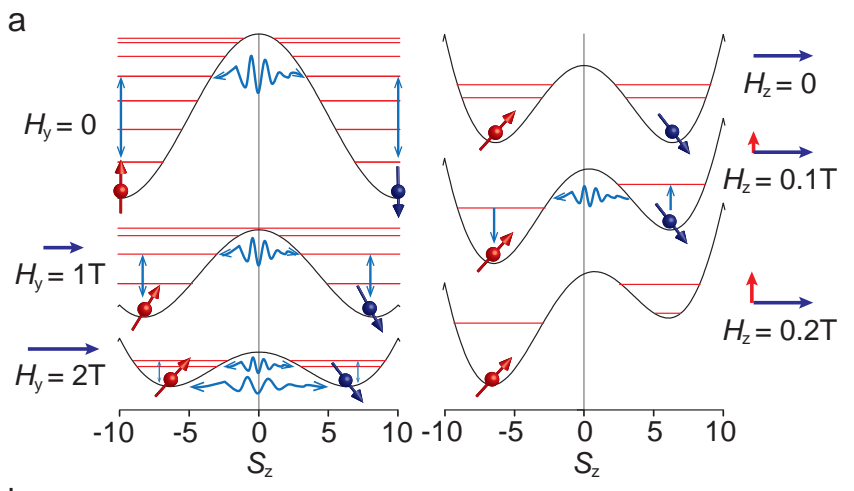

b

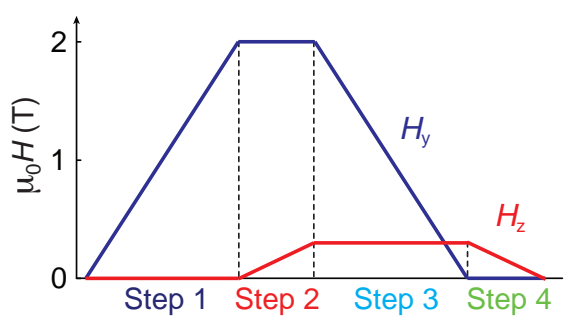

c

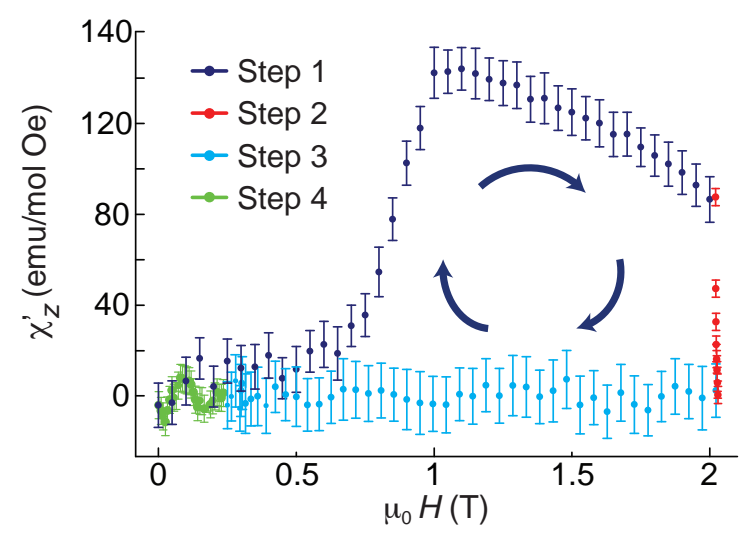

FIG. 2. Susceptibility of the quantum MM during the Landauer erasure. (a) Four-step sequence of magnetic fields $H_{y}$ (blue) and $H_{z}$ (red) inducing the erasure process. The first step $\left(H_{y}: 0 \rightarrow 2 \mathrm{~T}\right)$ corresponds to the bit erasure and the remaining three steps correspond to its reset. (b) Magnetic energy of a $\mathrm{Fe}_{8} \mathrm{MM}$ that is first subject to a transverse magnetic field $H_{y}$ of increasing strength (left, step 1) and then to a longitudinal magnetic field $H_{z}$ (right, step 2). Thick solid lines show the classical potential landscape while thin horizontal lines are the quantum energy levels determined via the numerical diagonalization of Eq. (1). $H_{y}$ keeps the symmetry of the potential intact but promotes quantum tunneling of the magnetization between quasi-degenerate $\pm S_{z}$ states, thus promoting the spin reversal; whereas $H_{z}$ introduces an energy bias between up and down spin states, thus increasing the magnetic polarization along the easy axis. (c) Real component of the longitudinal magnetic ac-susceptibility, $\chi_{z}^{\prime}$, as a function of the vector magnetic field's modulus, measured at $1 \mathrm{~K}$ and a frequency of $333 \mathrm{~Hz}$. The sequence of steps corresponds to the one in (a). 
By contrast, a magnetic field $H_{z}$ parallel to the easy axis favours either of the two eigenstates $S_{z}= \pm 10$, i.e., by increasing the "up" or "down" polarizations it selects states '0' or ' 1 '.

To perform the experiment, we first align our reference frame to the principal magnetic axes of crystal (see Appendix B) and then apply the sequence of magnetic fields depicted in Fig. 2(b) - comparable to that proposed in Ref. 5 for classical magnets. In step 1, the magnetic field along the medium axis of the MM $\left(H_{y}\right)$ is ramped up to $2 \mathrm{~T}$ and the spin states are mixed so that the bit is erased. In step $2, H_{z}$ is ramped up to $210 \mathrm{mT}$ in the constant $H_{y}$ field to reset all molecular spins in the "up" state. In steps 3 and 4, both magnetic field components are returned to zero, completing the bit storage process.

Throughout the protocol, the complex ac-susceptibility $\chi_{z}=\chi_{z}^{\prime}+i \chi_{z}^{\prime \prime}$ along the easy axis is measured with an inductive susceptometer (see Appendix A for details). $\chi_{z}$ is proportional to the derivative of the magnetization $\partial M / \partial H_{\mathrm{ac}}$ and a function of the temperature $T$, frequency $\omega$ of the ac-field $H_{\mathrm{ac}}$ and magnetic field vector $\boldsymbol{H}$. The magnetization and work can be derived by integrating $\chi_{z}^{\prime}$ once and twice with respect to magnetic field, respectively. The work $W$ obtained in this manner quantifies the entropy produced during the erasure and measures how reversible the reset operation is. ${ }^{5,15}$

The measurements are performed at $T=1 \mathrm{~K}$ and $\omega / 2 \pi=333 \mathrm{~Hz}$. This temperature is low enough to store the spins for minutes at zero field, ${ }^{18}$ and high enough to have them relaxing within hundreds of nanoseconds when in a transverse field. Furthermore, this temperature is well above the dipolar ferromagnetic ordering temperature $T_{\mathrm{C}}=0.6 \mathrm{~K}$ of $\mathrm{Fe}_{8} .{ }^{16}$ Therefore, at zero bias the molecular spins orient randomly along the anisotropy axis.

Results for the real component of the susceptibility $\chi_{z}^{\prime}$, are shown in Fig. 2(c). In step $1, \chi_{z}^{\prime}$, initially zero, steeply increases at $H_{y} \approx 0.6 \mathrm{~T}$, reaches a peak at $H_{y} \approx 1 \mathrm{~T}$ and slowly decreases up to $H_{y}=2 \mathrm{~T}$. In step $2, \chi_{z}^{\prime}$ sharply drops and reaches zero at $H_{z} \approx 0.19 \mathrm{~T}$. Upon retracting the fields in steps 3 and $4, \chi_{z}^{\prime}$ remains substantially zero. This behavior can be understood as follows: At the beginning of step 1, all spins are frozen in one of the potential wells, thus the spin register consists of an equal number of '0's and '1's. Upon ramping up $H_{y}$, the spin relaxation time $\tau_{\text {rel }}$ decreases (Fig. 2(b)). As soon as $\tau_{\text {rel }} \lesssim 1 / \omega$ the spins can follow the oscillations of $H_{\mathrm{ac}}$, $\chi_{z}^{\prime}$ peaks and the erasure begins. Further increase of $H_{y}$ determines a decrease of $\chi_{z}$ and reflects the spin wavefunctions delocalization, i.e. superposition of different $\pm S_{z}$ states, over the two potential wells. For $H_{y}=2 \mathrm{~T}$, approximately $20 \%$ of the molecules in the crystal have fully delocalized spin ground states (see Appendix B). In step 2, $\chi_{z}^{\prime}$ decreases for increasing spin polarization, vanishing when the magnetization $M_{z}$ along the anisotropy axis is saturated. At this point, the spins are initialized in the "up" configuration, that is, the molecular bits are reset to state ' 1 '. In step 3 , the admixing field is ramped a

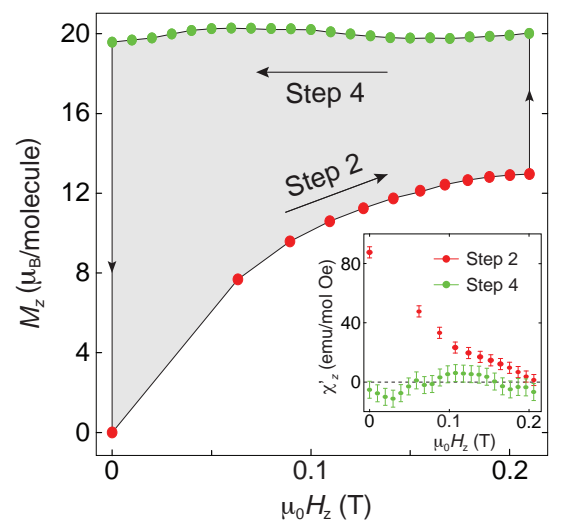

b

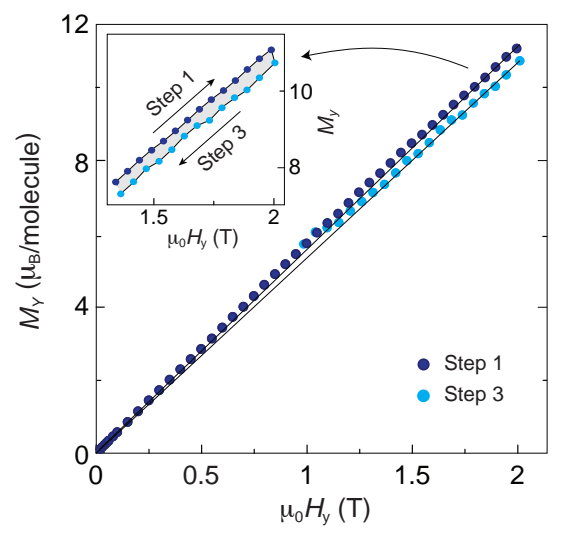

FIG. 3. Total bit reset work. (a) Longitudinal magnetization per molecule $M_{z}$ extracted by integrating $\chi_{z}^{\prime}$ of steps 2 and 4 (zoom-in from Fig. 2(c) in the inset) with respect to $H_{z}$. During step 2, $M_{z}$ increases for increasing $H_{z}$ before flattening out at about $13 \mu_{B}$. Upon retracting $H_{y}$ in step $3, M_{z}$ increases up to about its maximum value of $20 \mu_{B}$ and remains approximately constant as $H_{z}$ is also ramped to zero in step 4. The shaded area corresponds to the work done on the system by $H_{z}$. (b) SQUID magnetization per molecule $M_{y}$ along the medium axis of the MM as a function of transverse field $H_{y}$ at $T=2 \mathrm{~K}$. The slope of step 1 is higher than that of step 3 due to the small applied bias $H_{z}$ field (fits are guidelines to the eye). The area enclosed by these two curves (shaded area in the inset) corresponds to the work done by $H_{y}$. Adding the work values from (a) and (b) yields the energy needed for resetting one bit of information.

down. The potential energy barrier is then restored and spin tunneling is gradually turned off. This causes the spins to remain frozen in the chosen configuration upon retraction of the bias polarizing field (step 4).

By integrating the measured $\chi_{z}^{\prime}$ with respect to $H_{z}$ (inset of Fig. 3(a)), the easy axis magnetization $M_{z}$ for steps 2 and 4 can be calculated (see Appendix A). The result is shown in Fig. 3(a), where the value on the $y$ axis has been displayed in $\mu_{B}$ per molecule. The area enclosed by the magnetization loop amounts to the work made by the external magnetic field onto a single MM. This yields the value $W_{2,4} \equiv W_{4}-W_{2}=(1.7 \pm 0.3) \cdot 10^{-16}$ $\mathrm{erg} /$ molecule, where the uncertainty corresponds to a $1 \sigma$ 
confidence interval (see Appendix $\mathrm{C}$ for the determination of molecules' number and Appendix D for the uncertainties). To this quantity, the work $W_{1,3}$ done by the $H_{y}$ in steps 1 and 3 needs to be added. This work cannot be reliably extracted from the measured medium-axis susceptibility as this falls below our detection limit due to the strong magnetic anisotropy $\chi_{y} \ll \chi_{z}$. . However, $\chi_{y}$ is approximately independent of $T$ and $\omega$ (see Appendix B). The transverse magnetization $M_{y}$ can therefore be measured, with a SQUID, at $T=2 \mathrm{~K}$. The results, recorded as $H_{y}$ is first ramped up to $2 \mathrm{~T}$ (step 1) and subsequently ramped down in a small bias longitudinal field $H_{z}=0.21 \mathrm{~T}$ (step 3), are shown in Figure 3(b). The net work is given by the difference between $W_{3}$ and $W_{1}$ and amounts to $W_{1,3} \equiv W_{3}-W_{1}=(-6 \pm 2) \cdot 10^{-17}$ $\mathrm{erg} / \mathrm{molecule}$. The work, $W$, required for the erasure of each bit is then the sum $W=W_{1,3}+W_{2,4}=(1.1 \pm$ $0.3) \cdot 10^{-16} \mathrm{erg} /$ molecule. Within the experimental uncertainty, this is equivalent to the theoretical Landauer limit at the experimental temperature of $1 \mathrm{~K}$, which equals $k_{\mathrm{B}} T \ln 2=0.9570 \cdot 10^{-16} \mathrm{erg}$.

We now discuss the dynamics of the erasure process as the second important aspect in determining the $W$. $\tau_{\text {rel }}$. Ac-susceptibility measurements allow estimating the magnetic relaxation time $\tau_{\text {rel }}$ or, alternatively said, the time the spin system takes to reach thermal equilibrium. In particular, the ratio $\chi_{z}^{\prime \prime} /\left(\omega \chi_{z}^{\prime}\right) \simeq \tau_{\text {rel }}{ }^{17}$ In Fig. $4(\mathrm{a})$ we show the evolution of $\tau_{\text {rel }}$ as a function of $H_{y}$ during step 1 in the range $0.7 \leq H_{y} \leq 1.15 \mathrm{~T}$ for which $\chi_{z}^{\prime \prime} \pm$ $\sigma_{\chi^{\prime \prime}} \geq 0$, inset of Fig. 4(a). We complement these data with $\tau_{\text {rel }}$ extracted from $\chi_{z}^{\prime}$ measurements as a function of temperature (see Appendix B). The relaxation time exponentially drops from $71.2 \mathrm{~s}$ at $H_{y}=0 \mathrm{~T}$ to $1.09 \mu \mathrm{s}$ at $H_{y}=1.7 \mathrm{~T}$. Extrapolation to $H_{y}=2 \mathrm{~T}$ leads to a relaxation time of $196 \mathrm{~ns}$. This time is to be interpreted as the longitudinal response time of the phonon bathand-molecule system upon a change in $H_{z}$ and fixes the limit up to which quasi-static operation is retained and unwanted (dissipative) hysteresis are avoided.

The product of the work and relaxation time, $W \cdot \tau_{\text {rel }}$, yields $2.31 \cdot 10^{-23} \mathrm{erg} / \mathrm{bit} \cdot \mathrm{s}$. This figure quantifies the overall energy-time cost of a computation and its value can be compared to that of other storage devices, operating at room temperature $(T \approx 300 \mathrm{~K})$. As shown in Fig. 4(b) (see Appendix E for the extended chart), the product $W \cdot \tau_{\text {rel }}$ for standard flip-flops - moderately fast but lossy - is $\sim 10^{-9} \mathrm{erg} / \mathrm{bit} \cdot \mathrm{s}$; the optical trap system in Ref. 14 - slow but efficient - attains $\sim 10^{-12}$ $\mathrm{erg} / \mathrm{bit} \cdot \mathrm{s} . \quad$ Increased performances $\left(\sim 10^{-19} \mathrm{erg} / \mathrm{bit} \cdot \mathrm{s}\right)$ over these two system is given by the recent GdFeCo laser-driven ferromagnetic element in Ref. 10 owing to its tens of ps operation time. The $\mathrm{Fe}_{8} \mathrm{MM}$ performs about $10^{4}$ times better than this system - reducing to 100 times when accounting for the lower operating temperature. This shows that quantum tunneling provides the key to achieve energetically efficient and fast classical operations.

It is interesting to compare this figure with an inde-

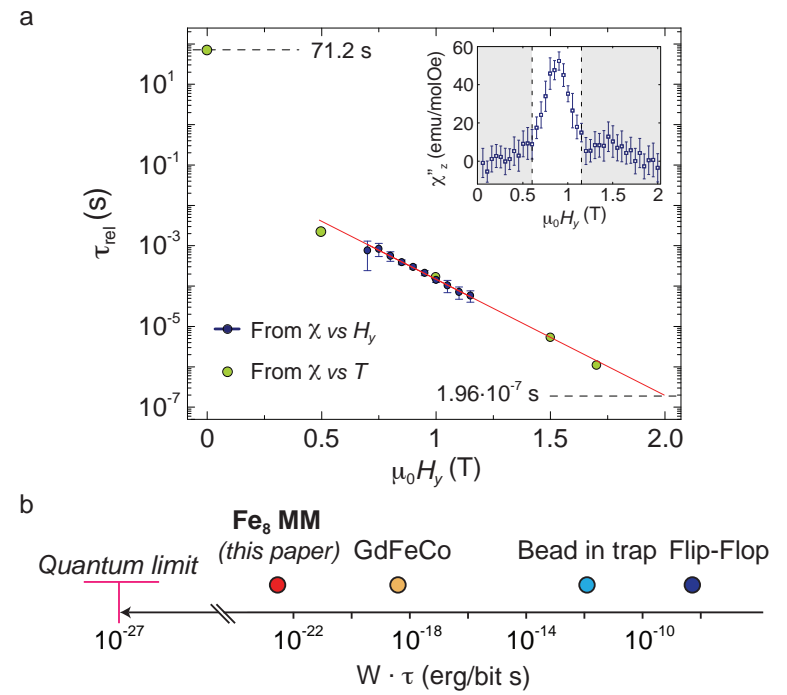

FIG. 4. Relaxation time and energy-time cost. (a) Evolution of the spin relaxation time as a function of $H_{y}$ during step 1 . The blue data points are obtained from $\chi_{z}^{\prime \prime}$ in the interval $0.7 \leq H_{y} \leq 1.15 \mathrm{~T}$ (inset). The green data points are extracted from temperature sweeps (see Appendix B). The relaxation time reaches $196 \mathrm{~ns}$ at $H_{y}=2 \mathrm{~T}$. This value sets the maximum speed up to which quasi-static operation is retained. (b) Chart comparing the energy-time cost of a storage operation performed with various systems at their respective operating temperature. $\mathrm{The}_{\mathrm{Fe}}$ in this study is the closest to the quantum limit.

pendent bound on the energy-time cost of a computation given by the Heisenberg uncertainty relation. ${ }^{22,23}$ According to it, the quantum evolution between two orthogonal, thus classically distinguishable, bit states split by an energy $E$ would take the minimal "relaxation" time $\tau_{\text {rel }}=\pi \hbar /(2 \Delta)$ called quantum speed limit ${ }^{24-26}$. From it, the limit $\pi \hbar / 2=1.65 \times 10^{-27} \mathrm{erg} / \mathrm{bit} \cdot \mathrm{s}$ is obtained as the ultimate trade-off between speed and energy cost.

We have shown that the Landauer limit applies also to the erasure of a quantum spin register. Quantum mechanics leaves its mark on the the speed, the energetic efficiency and the nature of the process. Contrary to the situation met with classical memories, the potential energy barrier does not need to be reduced to zero in order to erase the bits, because tunneling provides a very fast shortcut for the spin reversal. As a consequence of its discrete energy level spectrum, each molecule behaves then as a truly two-level system ${ }^{28}$ and the entropy production and work remain very close to $\Delta S \simeq k_{\mathrm{B}} \ln 2$ and $W \simeq k_{\mathrm{B}} T \ln 2$, respectively. In other words, the erasure can be energetically as efficient as allowed by the Landauer limit or, equivalently, by the second principle of thermodynamics. The erasure is also qualitatively different from its classical analogue. The wave functions of the two bit states, and not just their populations, are continuously shaped along the process from a delocalized configuration back to a pure spin projection $+S$. Given the generality of these operations, we expect that this 
result remains valid for a variety of realizations. Our findings would then suggest that the Landauer limit governs also the initialization of spin qubits from arbitrary states and provide a framework to further explore the thermodynamics of quantum information.

The research reported here was supported by an advanced ERC grant (Mols@Mols). We also acknowledge financial support by the Dutch Organization for Fundamental research (NWO/FOM). EB acknowledges funds from the EU FP7 program through the project 618082 ACMOL. FL acknowledges the Spanish MINECO (grant MAT2015-68204-R), the Gobierno de Aragón (grant E98MOLCHIP) and the European Union (COST 15128 Molecular Spintronics project). R.G. especially thanks Prof. Dr. Luca Gammaitoni for inspiring discussions. *r.gaudenzi@tudelft.nl

1 Landauer, R. Irreversibility and heat generation in the computing process. IBM Journal of Research and Development 5, 183-191 (1961).

2 Bennett, C. H. The thermodynamics of computation - a review. International Journal of Theoretical Physics 21, 905-940 (1982).

3 Bennett, C. H. Notes on the history of reversible computation. IBM Journal of Research and Development 32, 16-23 (1988).

4 Leff, H. \& Rex, A. Maxwell's demon: Information, entropy, computing. A Hilger and Princeton Univ. Press, Europe/USA (1990).

${ }^{5}$ Lambson, B., Carlton, D. \& Bokor, J. Exploring the thermodynamic limits of computation in integrated systems: Magnetic memory, nanomagnetic logic, and the Landauer limit. Phys. Rev. Lett. 107, 010604 (2011).

${ }^{6}$ Gammaitoni, L., Chiuchiu, D., Madami, M. \& Carlotti, G. Towards zero-power ict. Nanotechnology 26, 222001 (2015).

7 Gerrits, T., van den Berg, H. A. M., Hohlfeld, J., Bar, L. \& Rasing, T. Ultrafast precessional magnetization reversal by picosecond magnetic field pulse shaping. Nature $\mathbf{4 1 8}$, 509-512 (2002).

8 Žutić, I., Fabian, J. \& Das Sarma, S. Spintronics: Fundamentals and applications. Rev. Mod. Phys. 76, 323-410 (2004).

9 Ostler, T. A. et al. Ultrafast heating as a sufficient stimulus for magnetization reversal in a ferrimagnet. Nature Communications 3, 666 (2012).

10 Yang, Y. et al. Ultrafast magnetization reversal by picosecond electrical pulses. arXiv preprint arXiv:1609.06392 (2016).

11 Lloyd, S. Quantum-mechanical Maxwell's demon. Phys. Rev. A 56, 3374-3382 (1997).

12 Lesovik, G. B., Lebedev, A. V., Sadovskyy, I. A., Suslov, M. V. \& Vinokur, V. M. H-theorem in quantum physics. Sci. Reports 6, 32815, 1-7 (2016).

13 Jun, Y., Gavrilov, M. \& Bechhoefer, J. High-precision test of landauer's principle in a feedback trap. Phys. Rev. Lett. 113, 190601 (2014).

14 Berut, A. et al. Experimental verification of landauer's principle linking information and thermodynamics. Nature 483, 187-U1500 (2012).

15 Hong, J., Lambson, B., Dhuey, S. \& Bokor, J. Experimental test of landauer's principle in single-bit operations on nanomagnetic memory bits. Science advances 2, 1501492 (2016).

16 Burzurí, E. et al. Magnetic dipolar ordering and quantum phase transition in an $\mathrm{fe}_{8}$ molecular magnet. Phys. Rev. Lett. 107, 097203 (2011).
17 Gatteschi, D., Sessoli, R. \& Villain, J. Molecular nanomagnets, vol. 5 (Oxford University Press on Demand, 2006).

18 Wernsdorfer, W. \& Sessoli, R. Quantum Phase Interference and Parity Effects in Magnetic Molecular Clusters. Science 284 133-135 (1999).

19 Sangregorio, C., Ohm, T., Paulsen, C., Sessoli, R. \& Gatteschi, D. Quantum tunneling of the magnetization in an iron cluster nanomagnet. Phys. Rev. Lett. 78, 4645-4648 (1997).

20 Burzurí, E. et al. Quantum interference oscillations of the superparamagnetic blocking in an $\mathrm{fe}_{8}$ molecular nanomagnet. Phys. Rev. Lett. 111, 057201 (2013).

${ }^{21}$ Luis, F., Bartolome, J. \& Fernandez, J. F. Resonant magnetic quantum tunneling through thermally activated states. Physical Review B 57, 505-513 (1998).

22 Margolus, N. \& Levitin, L. B. The maximum speed of dynamical evolution. Physica D 120, 188-195 (1998).

23 Lloyd, S. Ultimate physical limits to computation. Nature 406, 1047-1054 (2000).

24 Aharonov, Y. \& Bohm, D. Time in the quantum theory and the uncertainty relation for time and energy. Phys. Rev. 122, 1649-1658 (1961).

25 Anandan, J. \& Aharonov, Y. Geometry of quantum evolution. Phys. Rev. Lett. 65, 1697-1700 (1990).

26 Deffner, S. \& Lutz, E. Energy-time uncertainty relation for driven quantum systems. Journal of Physics aMathematical and Theoretical 46 (2013).

27 Campbell, S. \& Deffner, S. Trade-off between speed and cost in shortcuts to adiabaticity. Physical Review Letters 118 (2017).

28 The population of levels above the ground doublet remains always below $2 \%$ 

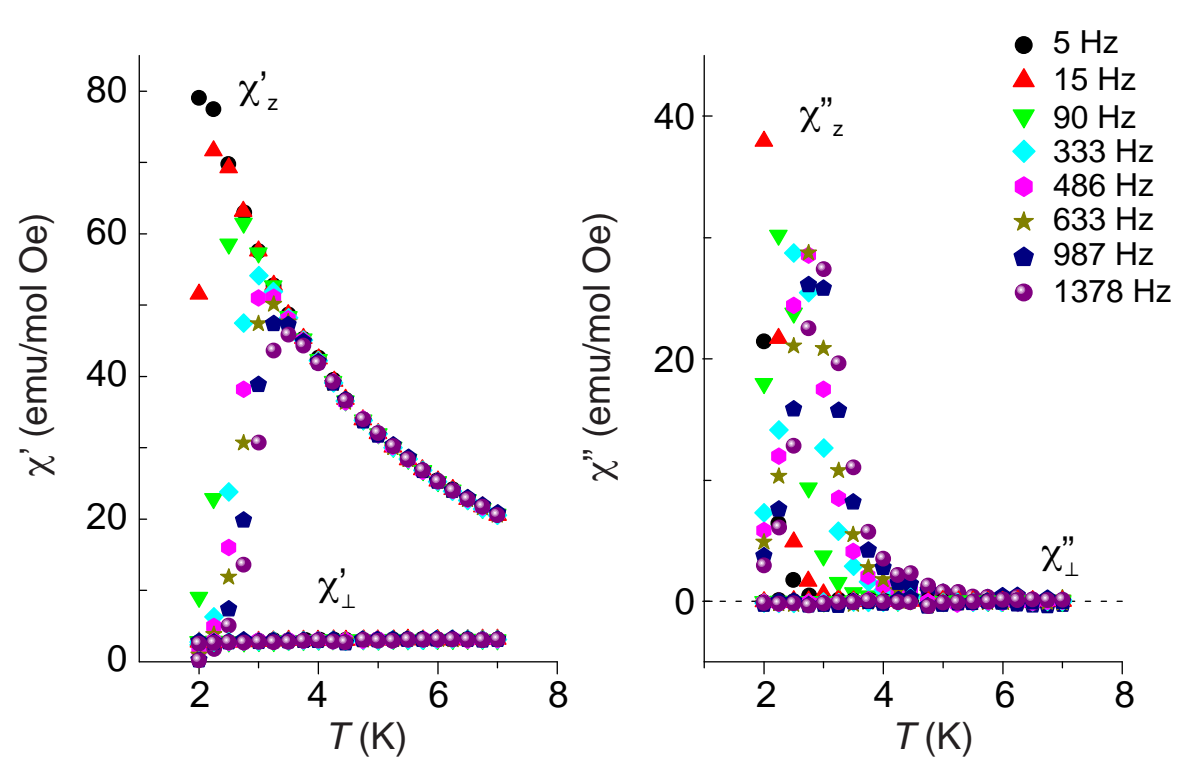

FIG. B.1. Temperature and frequency characterization. (a) Real (left) and imaginary (right) part of the susceptibility as a function of $T$ and indicated frequency $\omega$ ranging from $5 \mathrm{~Hz}$ to $1378 \mathrm{~Hz}$. The frequency-dependent departure from equilibrium signals the expected superparamagnetic behaviour.

\section{Appendix A: Methods}

Susceptibility measurements An ac-susceptometer thermally anchored to the mixing chamber of a dilution refrigerator in combination with a $3 \mathrm{D}$ vector magnet $\left(9 \mathrm{~T}, 1 \mathrm{~T}, 1 \mathrm{~T}, 0.001^{\circ}\right.$ accuracy) is used to measure the erasure-storage protocol. The complex susceptibility $\chi(T, \omega)=\chi^{\prime}(T, \omega)+i \chi^{\prime \prime}(T, \omega)$ is measured with a standard lock-in technique with an ac excitation magnetic field of amplitude $H_{a c}=0.01$ Oe parallel to the common easy axis of the molecules.

Magnetization measurements Magnetization is measured with a commercial SQUID magnetometer $(T \geq 1.8 \mathrm{~K})$ equipped with a rotating stage and an ac susceptibility option.

Calculations of magnetization and work The easy-axis magnetization $M_{z}$ is obtained from the susceptibility $\chi_{z}^{\prime}$ by making use of the integral:

$$
M_{z}=\int \chi_{z}^{\prime} d H_{z}
$$

The works done by $H_{z}$ (steps 2 and 4 ) and $H_{y}$ (steps 1 and 3) are calculated by performing an analogous integration on the resulting $M_{z}$ and $M_{y}$, respectively:

$$
W_{2,4}=\oint M_{z} d H_{z}, \quad W_{1,3}=\oint M_{y} d H_{y}
$$

These correspond to the loop shaded areas in Fig. 3 (a) and (b).

\section{Appendix B: Characterisation in temperature and transverse field}

This section contains details on: (i) the characterisation of the superparamagnetic behaviour of the MM crystal in temperature and frequency; (ii) the extraction of the relaxation time as a function of selected $H_{y}$ fields; (iii) the determination of the crystal's magnetic easy and medium axes orientation with respect to the laboratory's reference system.

(i) Temperature and frequency In Fig. B.1, the real (in-phase) $\chi^{\prime}(T, \omega)$ and imaginary (out-of-phase) $\chi^{\prime \prime}(T, \omega)$ components of the ac-susceptibility are shown as a function of temperature for the indicated frequencies in zero magnetic field. For each component, the longitudinal $\chi_{z}$ and transverse $\chi_{\perp}$ parts are plotted. For a fixed frequency, 
decreasing the temperature results in the increase of $\chi_{z}^{\prime}$ accompanied by a constant $\chi_{z}^{\prime \prime} \approx 0$. This is the typical behaviour of a standard paramagnet, where the absence of an out-of-phase response signals equilibrium and a fast relaxation time $\tau_{\text {rel }} \ll 1 / \omega$. However, as a frequency-dependent temperature is reached, $\chi_{z}^{\prime}$ starts dropping to zero while $\chi_{z}^{\prime \prime}$ exhibits a peak. This temperature corresponds to the so-called blocking temperature, $T_{b}$, and it is characterised by a spin relaxation time $\tau_{\text {rel }} \simeq 1 / \omega$. For $T<T_{b}$, the spin of the MM is increasingly driven out-of-equilibrium and $\tau_{\text {rel }}$ further increases. The observed behaviour is a fingerprint of the superparamagnetism expected in a MM, where the potential barrier prevents fast spin relaxation at sufficiently low temperatures. The small temperature- and frequency-independent $\chi_{\perp}^{\prime}$ and zero $\chi_{\perp}^{\prime \prime}$ further signal the strong spin polarisation along the easy-axis and negligible transverse (hard-plane) spin projection. 
(ii) Transverse magnetic field To extract the relaxation time data-points shown in Fig. 4 (and labelled " $\chi$ vs $T$ data"), we use temperature-dependent complex susceptibility measurements for the different $H_{y}$ fields at the frequency $\omega=333 \mathrm{~Hz}$. Plotting the ratio $\chi_{z}^{\prime \prime} / \omega \chi_{z}^{\prime}=\tau_{r e l}$ as a function of the inverse temperature results in Fig. B.2. At high temperatures, the relaxation time behaves according to Arrhenius' law $\log \tau_{\text {rel }}=U_{\text {eff }} / T+\tau_{0}$ with the effective barrier $U_{\text {eff }}\left(H_{y}\right)$ obtained by fitting the temperature-dependent part of the curves. Extrapolation of the fit to $T=1 \mathrm{~K}$, yields $\tau_{r e l}\left(H_{y} ; T=1 \mathrm{~K}\right)$ for the selected fields.

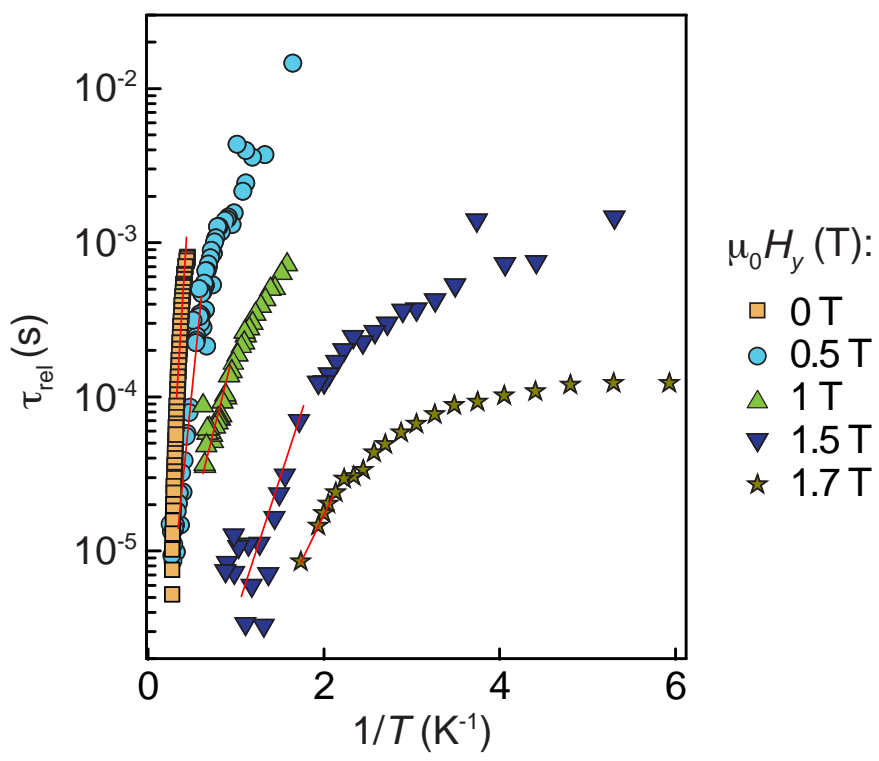

FIG. B.2. Relaxation time for different transverse fields. (a) $\tau_{\text {rel }}$, obtained from $\chi_{z}^{\prime \prime} / \omega \chi_{z}^{\prime}$, as a function of the reciprocal temperature. Fitting of the temperature-dependent side allows to obtain the desired $\tau_{\text {rel }}\left(H_{y}\right)$ at $T=1 \mathrm{~K}$ by extrapolation.

(iii) Alignment to principal axes In this subsection we describe the procedure used for finding the accurate orientation of the principal axes of the MM with respect to the $X, Y$ and $Z$ axes of the vector magnet. Provided this orientation is approximately known, the crystal is placed in the susceptometer with its easy, medium and hard axes about the $Y, Z$ and $X$-axis of the magnet, respectively. Measurements are executed at $T=3 \mathrm{~K}$ and $\omega=1333$ Hz. Under these conditions, the susceptibility is close to equilibrium (see Fig. B.1) and thus strongly dependent on the magnetic field orientation. The first operation consists of rotating the magnetic field $\mu_{0}|H|=0.1 \mathrm{~T}$ on the $X Z$-plane by fixing $\phi=0$ and sweeping $\theta$. As Fig. B.3(a) shows, $\chi_{z}^{\prime}$ exhibits a maximum (minimum), signalling a condition relatively closer to (further from) equilibrium. In correspondence of the maximum, for $\theta=5.70^{\circ}$, the magnetic field crosses the hard plane, while it is closest to the easy axis in correspondence of the minimum. An analogous operation is conducted on the $X Y$-plane (Fig. B.3(b)), where the crossing with the hard plane occurs for $\phi=22.72^{\circ}$. The cross product between the two hard plane vectors yields an easy axis with angular coordinates $\theta=87.79^{\circ}$ and $\phi=112.72^{\circ}$. This axis is hereafter labelled by the subscript $z$. A confirmation of the accurate orientation of this axis is shown in Fig. B.3(c). Sweeping the magnetic field along it gives rise to a peak at 0.22 $\mathrm{T}(0.44 \mathrm{~T})$, in correspondence of the resonance between the spin eigenstates $S_{z}=10$ and $S_{z}=-9\left(S_{z}=10\right.$ and $\left.S_{z}=-8\right)$ expected at $B_{n}=\frac{D}{g \mu_{\mathrm{B}}} n=0.219 \mathrm{~T} \times n$, for $n=1(2)$.

At this point, the magnetic field is swept on the hard plane for $\theta=90^{\circ}$ and $\phi=22.72^{\circ}$ (Fig. B.4). The observed oscillatory behaviour in $\chi_{z}^{\prime}$, with minima at the indicated fields, is in accordance with the characteristic quantum interference pattern (see Ref. [17] of the main text) occurring in the proximity to the hard axis - labelled by $x$ hereafter. A $90^{\circ}$-shift from this axis along the hard plane fixes the medium axis - labelled by $y$ - and concludes the orientation procedure.

\section{Appendix C: Determination of the number of molecules}

We have determined the number of molecules (bits) in the crystal with two independent methods. The first and most straightforward is that of dividing the weight of the crystal, $m=0.411 \mathrm{mg}$, by the molecular weight, $P_{\mathrm{m}}=2262.45 \mathrm{~g} / \mathrm{mol}$ and multiply by the Avogadro constant. This yields a number of molecules $N=1.094 \cdot 10^{17}$. 
a

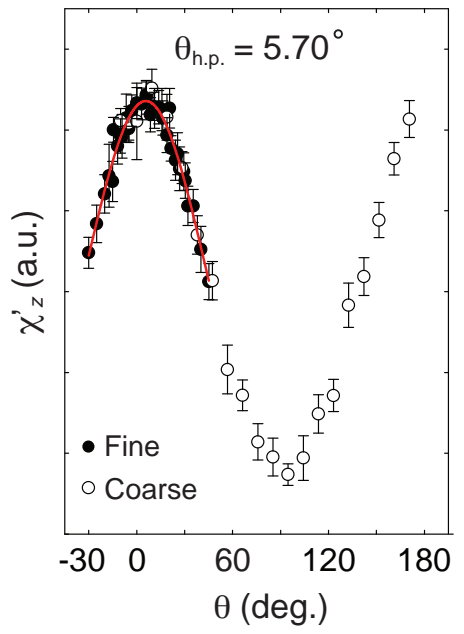

b

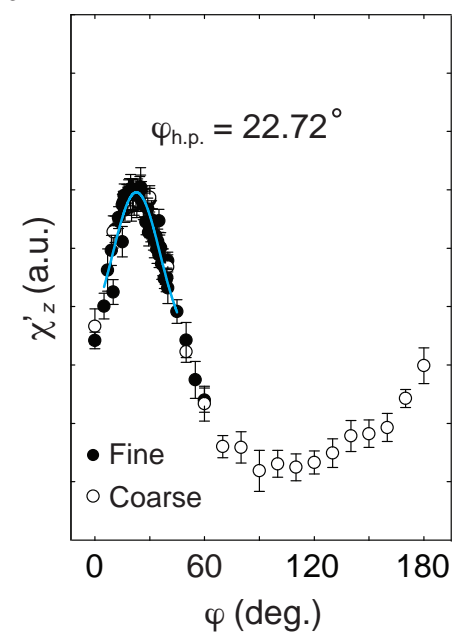

C

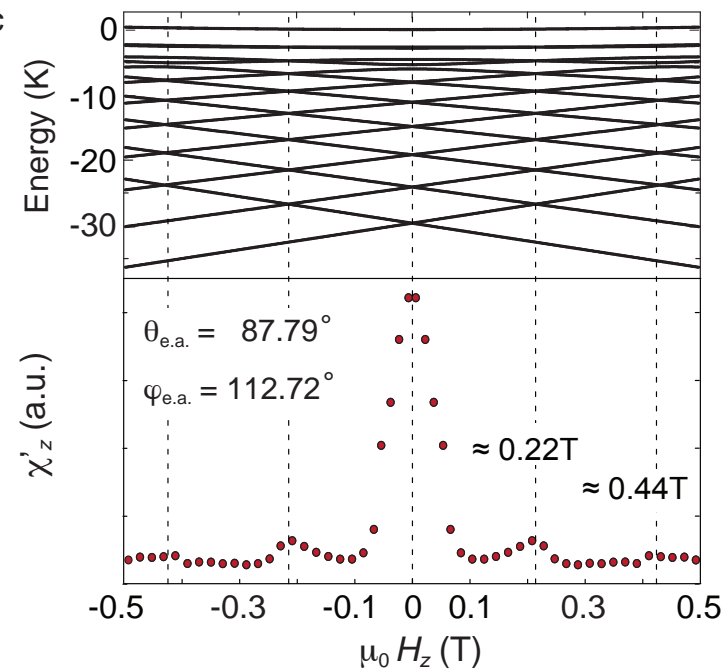

FIG. B.3. Determination of the easy axis. (a) $\chi_{z}^{\prime}$ as a function of angle $\theta$ for fixed $\phi=0^{\circ}$ ( $X Z$-plane). (b) Same as (a) for fixed $\theta=90^{\circ}$ ( $X Y$-plane). Maxima signal the two crossings with the hard plane from which the orientation of the easy axis is obtained. (c) $\chi_{z}^{\prime}$ as a function of magnetic field intensity along the easy axis (labelled by the subscript $z$ ). The three peaks signal the expected magnetic level crossings.
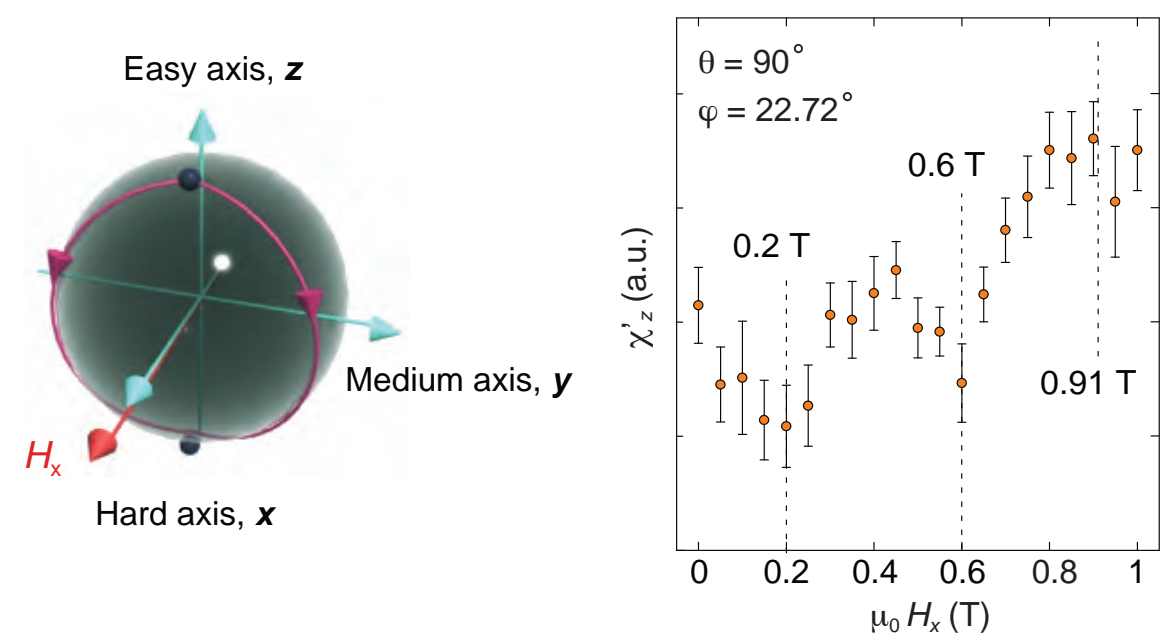

FIG. B.4. Quantum interference pattern along the hard axis. $\chi_{z}^{\prime}$ as a function of magnetic field intensity along the hard axis (labelled by the subscript $x$ ). Oscillations signal the expected quantum interference pattern with minima at the indicated fields.

The second method takes advantage of the fact that each molecule has a definite spin $S=10\left(20 \mu_{\mathrm{B}}\right)$. By measuring the saturation magnetization, $M_{s}$, of the crystal in the SQUID setup and dividing by the spin of the single molecule yields:

$$
N=\frac{M_{s}(\mathrm{emu})}{20 \mu_{\mathrm{B}}} 5.1883 \cdot 10^{20} \mu_{\mathrm{B}} / \mathrm{emu}
$$

Provided $M_{s}=(2.029 \pm 0.006) \cdot 10^{-2} \mathrm{emu}($ Fig. C.5 $), N=(1.09392 \pm 0.00326) \cdot 10^{17}$. 

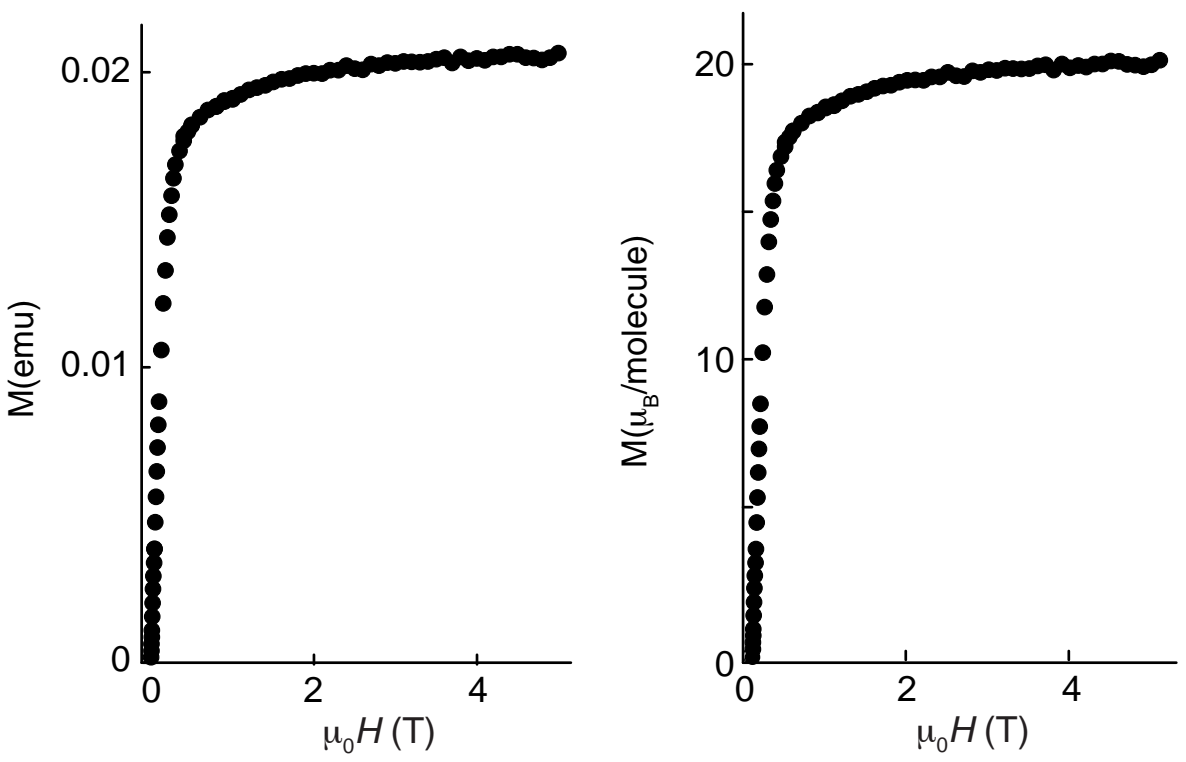

FIG. C.5. Saturation of the magnetization. (a) Raw magnetization $M$ (emu) measured in the SQUID as a function of magnetic field. (b) Scaled magnetization $M\left(\mu_{\mathrm{B}} /\right.$ molecule) obtained normalizing the raw magnetization to the single-molecule value of $20 \mu_{\mathrm{B}}$. The ratio between the two quantities yields the indicated estimate for the number of molecules in the crystal.

\section{Appendix D: Quantum nature of the Landauer process: wave functions and spin relaxation}

The states and the magnetic response of $\mathrm{Fe}_{8}$ MMs depend on interactions that are intrinsic to each individual molecule, such as the magnetic anisotropy and the Zeeman interaction with an external magnetic field, described by the spin Hamiltonian 1, as well as on couplings to the 'surrounding world', chiefly to other spins in the crystal and to lattice vibrations.
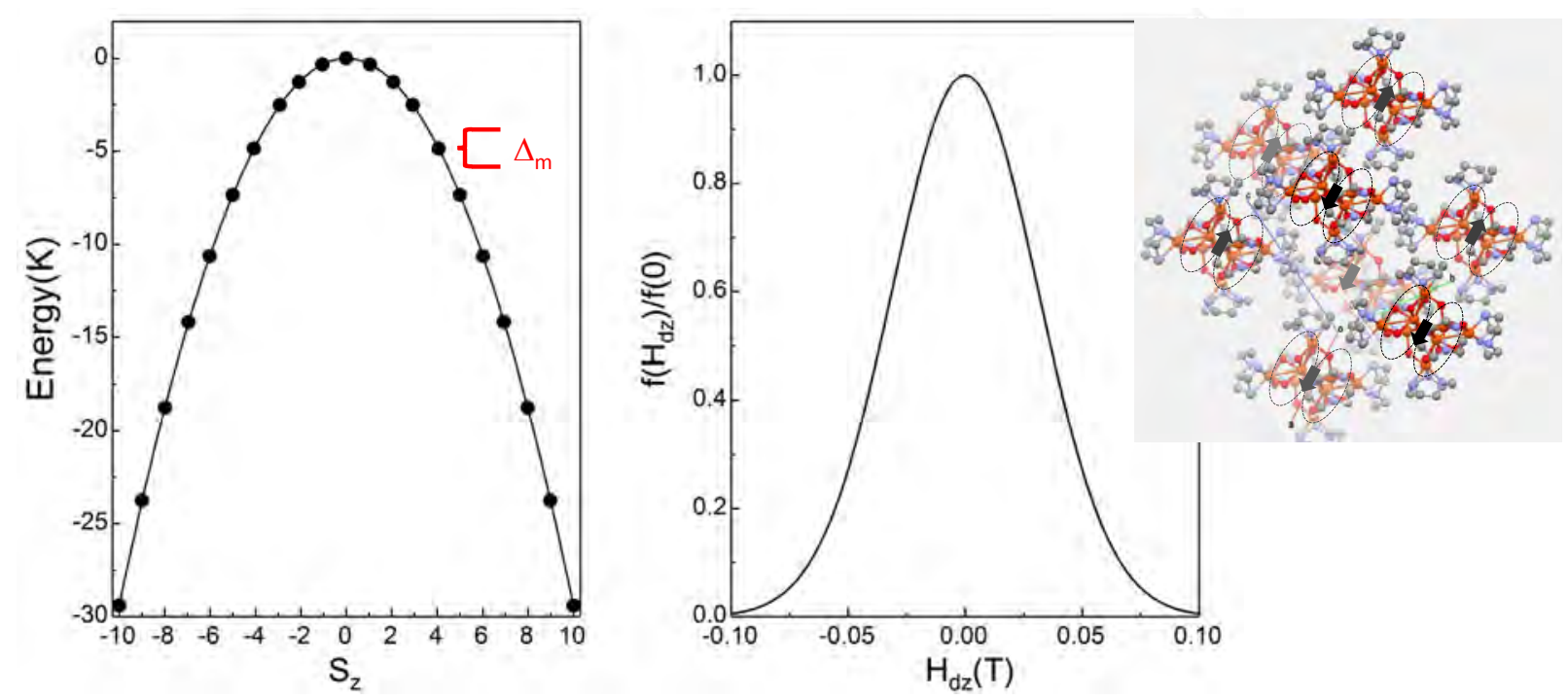

FIG. D.6. Magnetic anisotropy vs dipole-dipole interactions. (a)Uniaxial magnetic anisotropy $-D S_{z}^{2}$ generates a zerofield splitting of levels associated with different spin projections $m$ along the anisotropy axis $z$ that resembles the well-known classical double-well potential. The ground state doublet is given by states with $m= \pm 10$, which encode the states ' 1 ' and ' 0 ' of a magnetic bit. Off-diagonal terms modify the classical potential landscape and introduce quantum tunneling between $|+m\rangle$ and $|-m\rangle . \Delta_{m}$ is the quantum tunneling splitting. (b) Distribution of bias dipolar fields $H_{\mathrm{d}, \mathrm{z}}$ in a magnetically unpolarized crystal of $\mathrm{Fe}_{8}$ (inset) at $H=0$. 
Longitudinal anisotropy terms, i.e., those including only powers of $S_{z}$, generate a zero-field splitting of the magnetic levels, associated with $S_{z}$ eigenstates $|m\rangle\left(S_{z}|m\rangle=m|m\rangle\right)$, and define the ground states (that we here associate to '0' and '1') as the maximum spin projections along the anisotropy axis $z$. Transverse anisotropy terms, which include powers of $S_{x}$ and $S_{y}$, modify this picture. In classical mechanics, any distortion from a perfect axial anisotropy modifies the double well potential and the activation barrier. In quantum mechanics, these terms mix different $|m\rangle$ states, introduce a finite probability of tunneling from $|+m\rangle$ to $|-m\rangle$, and split the initially degenerate states by a quantum tunneling splitting $\Delta_{m}$. These effects are illustrated in Fig. D.6a.

Each molecular spin generates a magnetic dipolar field $\boldsymbol{H}_{\mathbf{d}}$ that acts on its neighbors. Because of the strong magnetic anisotropy, its main effect is a bias $\xi_{m} \equiv 2 g \mu_{\mathrm{B}} m H_{\mathrm{d}, \mathrm{z}}$ associated with the component $H_{\mathrm{d}, \mathrm{z}}$ along $z$. Eventually, these interactions lead to long range magnetic order below a critical temperature $T_{\mathrm{c}}\left(\simeq 0.6 \mathrm{~K}\right.$ for Fe $\mathrm{Fe}_{8}{ }^{16} \mathrm{Above}_{\mathrm{c}}$ and for a magnetically unpolarized crystal (i.e., when $H_{z}=0$, as in step 1 of the Landauer erasure), dipolar interactions give rise to a close to Gaussian distribution in $H_{\mathrm{d}, \mathrm{z}}$, with a width $\sigma \simeq 31 \mathrm{mT}$, as shown in Fig. D.6a.
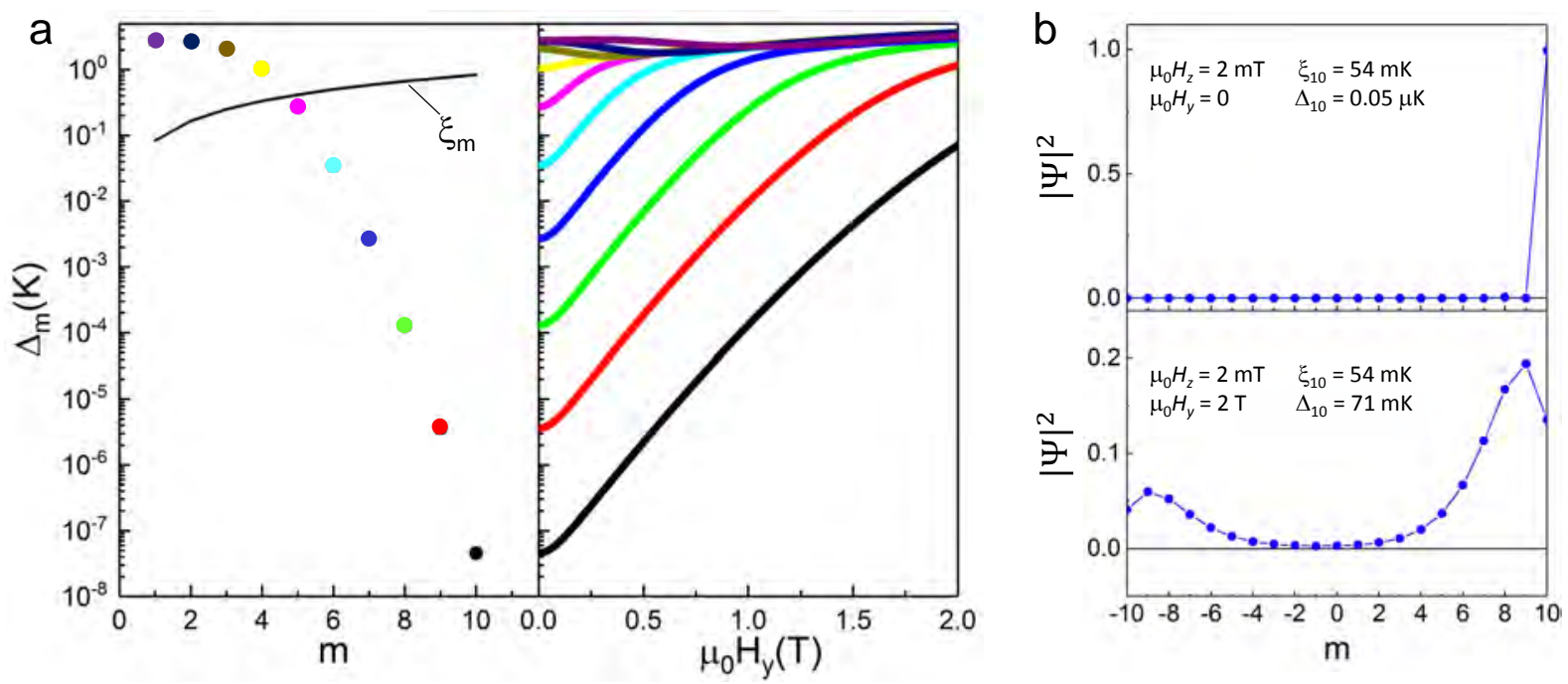

FIG. D.7. Quantum spin tunneling and wave-functions. (a) Quantum tunneling splittings for all level doublets at $H_{y}=0$ (left) and as a function of $H_{y}$, as in step 1 of the Landauer erasure process. The solid line shows the bias $\xi_{m} \equiv 2 g \mu_{\mathrm{B}} m H_{\mathrm{d}, \mathrm{z}}$ generated by a field $H_{\mathrm{d}, \mathrm{z}}=31 \mathrm{mT}$ (width of the dipolar field distribution at zero field). (b) Wave function of the ground state of $\mathrm{Fe}_{8}$ for $H_{z}=2 \mathrm{mT}$ and two values of $H_{y}$ corresponding to the beginning and the end of step 1, respectively.

The relative strength of $\Delta_{m}$ and $\xi_{m}$ determines the shape of the wave functions, being close to pure spin projections for $\Delta_{m} \ll \xi_{m}$ and becoming delocalized over different $|m\rangle$ states otherwise (see Fig. D.7b). This situation can be tuned externally, via the application of a magnetic field along the medium axis that, as shown in Fig. D.7, enhances $\Delta_{m}$ of all levels. 

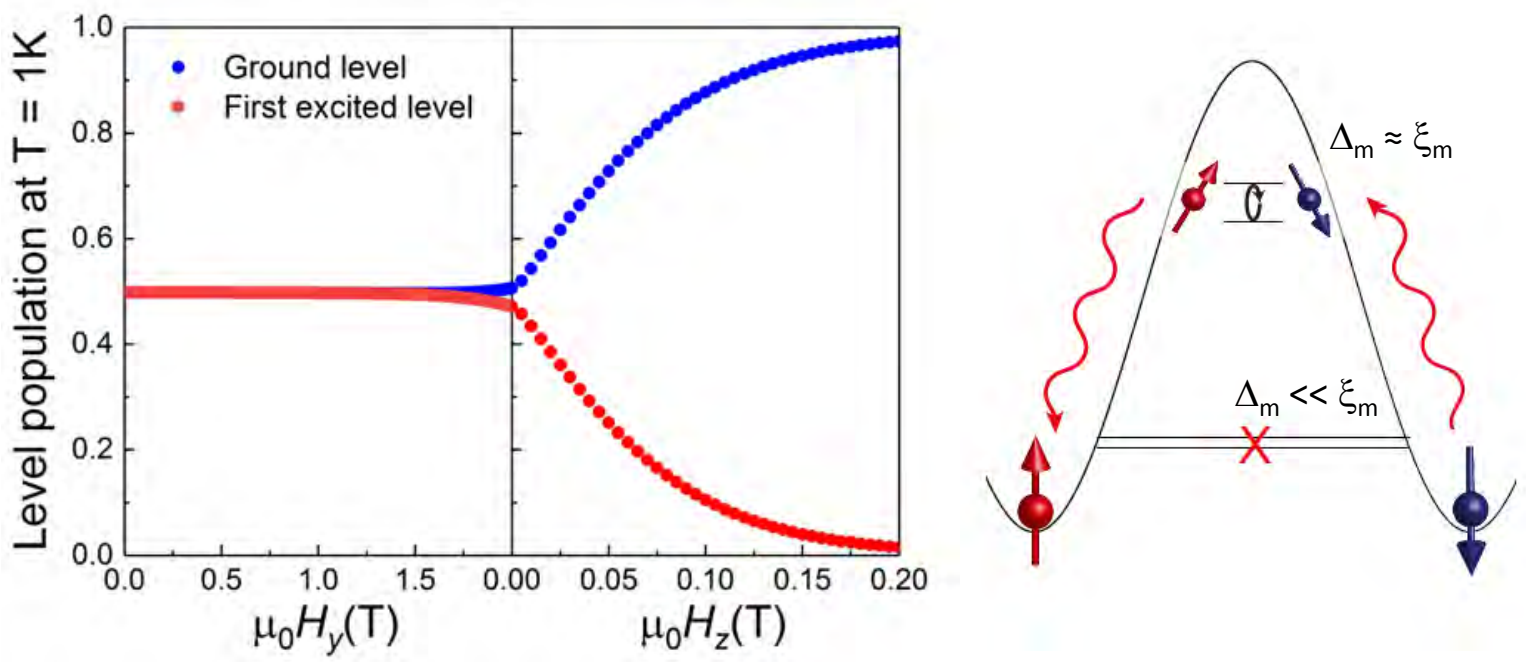

FIG. D.8. Phonons: thermal populations and spin relaxation. (a) Equilibrium populations of the two lowest lying magnetic states of a $\mathrm{Fe}_{8}$ at $T=1 \mathrm{~K}$ calculated under the conditions applicable to steps 1 (left) and 2 (right) of the Landauer erasure process. (b) Illustrative scheme of spin-lattice relaxation in $\mathrm{Fe}_{8}$ at finite temperatures.

The coupling of the spins to lattice vibration modes, i.e. phonons, enables the attainment of thermal equilibrium. The populations of the two lowest lying levels of $\mathrm{Fe}_{8}$ are shown in Fig. D.8a. In step 1, they are very close to each other and to $1 / 2$ (the population of excited states remains always below $2 \%$ ), thus entropy is close to $k_{\mathrm{B}} \ln 2$ per spin, whereas in step 2 the external bias drives the system to a well defined ground state (state ' 1 '). Therefore, each $\mathrm{Fe}_{8}$ provides a close to ideal realization of a two-level system throughout the erasure process.

Spin relaxation combines tunneling with excitations induced by the spin-phonon coupling and it is illustrated in Fig. D.8b. At finite temperatures, spin reversal can take place via the absorption of phonons to reach states having $\Delta_{m}$ not much smaller than $\xi_{m}$, followed by tunneling to the opposite side of the potential barrier and a de-excitation, emitting a phonon to the lattice, to the ground state. These events become exponentially more rare as $T$ decreases, thus leading to very long $\tau_{\text {rel }} \gtrsim 100 \mathrm{~s}$ at $T=1 \mathrm{~K}$ and, therefore, to magnetic memory. The situation can be changed by increasing $H_{y}$. Lower and lower levels then fulfil $\Delta_{m} \gtrsim \xi_{m}$ and $\tau_{\text {reldecreases. }}$

The discussion of the relaxation process can be made quantitative, as follows. Transitions between different energy spin states are induced by absorption and emission of resonant phonons (cite Luis1998, Chudnovsky1997,Loss2000). Here, we have estimated the relaxation rate and the dynamical susceptibility by numerically solving the following quantum master equation

$$
\frac{d P_{n}(t)}{d t}=\sum_{n^{\prime} \neq n} \gamma_{n \leftarrow n^{\prime}} P_{n^{\prime}}-\sum_{n^{\prime} \neq n} \gamma_{n^{\prime} \leftarrow n} P_{n}
$$

where $P_{n}$ and $P_{n^{\prime}}$ are, respectively, the (time-dependent) populations of the spin Hamiltonian eigenstates $\left|\psi_{n}\right\rangle$ and $\left|\psi_{n}\right\rangle$, while $\gamma_{n \leftarrow n^{\prime}}$ and $\gamma_{n^{\prime} \leftarrow n}$ are phonon-induced transitions between them, calculated using Fermi's golden rule. Further details can be found in 21.

At sufficiently low temperatures, direct tunneling via the ground state, not induced by phonons, becomes relevant and often provides the dominant spin relaxation channel. Both the nature the rate $\Gamma_{\text {tun }}$ of this process depend on details of the spin interaction with its environment, i.e. phonons, nuclear spins, other MMs as well as on the tunnel splitting $\Delta$. However, in most situations the following expression provides a suitable description(cite Prokof'ev1998,Fernandez2005)

$$
\Gamma_{\text {tun }}=\eta \Delta^{2} f\left(\xi=-2 g \mu_{\mathrm{B}} H_{z} S\right)
$$

where $\eta$ is a constant. Equation (D2) reflects the fact, discussed above (cf Fig. D.8), that only MMs with sufficiently low net bias are able to tunnel at any time. 
In this work, we describe our experiments with the combination of spin-phonon rates, derived from Eq. (D1), and tunneling rates, obtained using Eq. (D2). The only two fitting parameters are the strength of the spin-phonon interaction and $\eta$
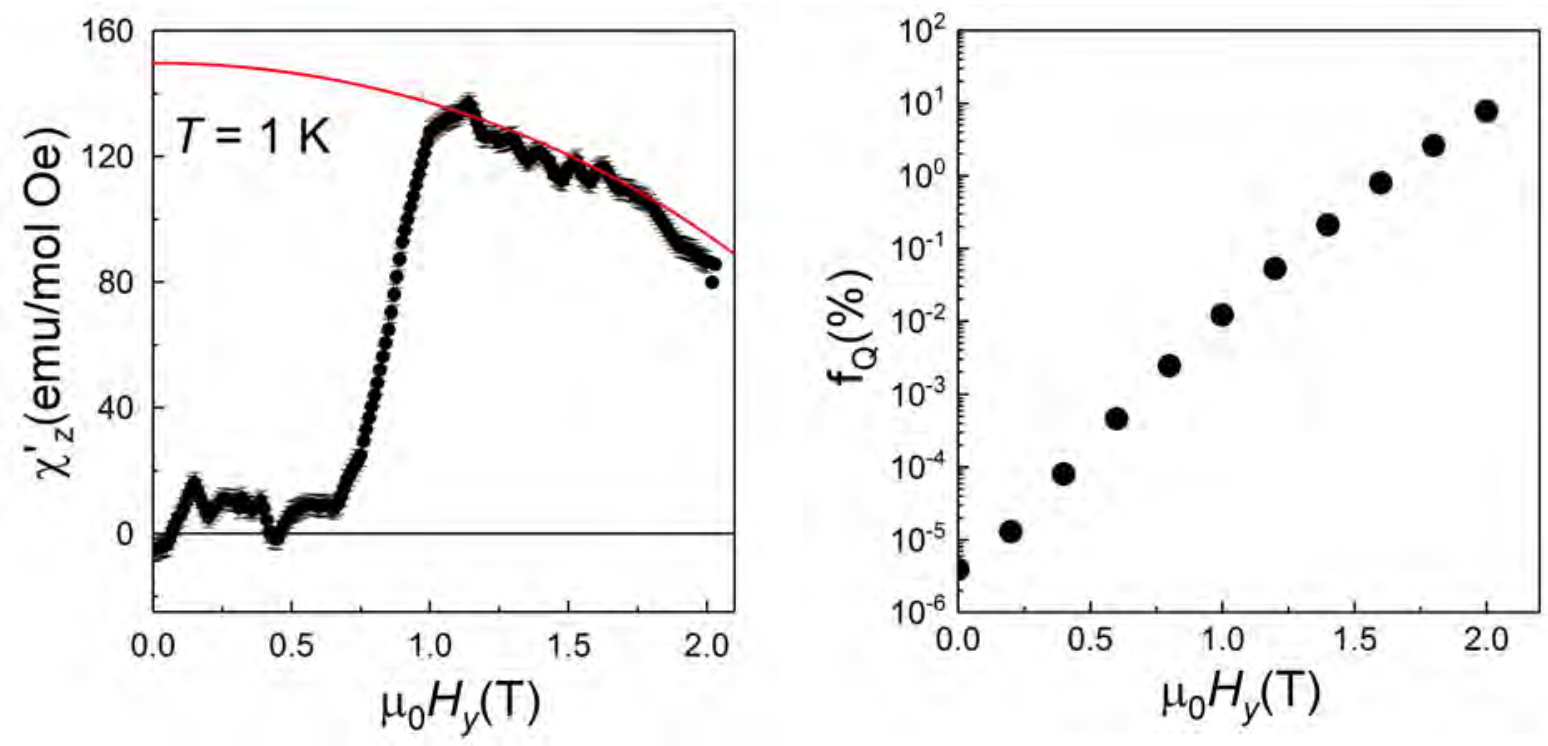

FIG. D.9. Fraction of "quantum molecules". (a) Dots: longitudinal ac susceptibility $\chi_{z}^{\prime}$ of $\mathrm{Fe}_{8}$ measured as a function of $H_{y}$ (step 1). For $H_{y} \gtrsim 1.1 \mathrm{~T}$ it corresponds to the equilibrium susceptibility $\chi_{z}$. Line: equilibrium susceptibility $\chi_{z}$ of Fe ${ }_{8}$ at $T=1 \mathrm{~K}$, calculated from the quantum eigenstates of the spin Hamiltonian (1). (b) Fraction of $\mathrm{Fe}_{8} \mathrm{MMs}_{\mathrm{s}}$ having $\Delta_{m} \geq \xi$ as a function of $H_{y}$ (step 1).

Finally, we discuss the nature of the spin ground state along the Landauer process. In step $1, H_{y}$ is ramped from 0 up to $2 \mathrm{~T}$. As we have just discussed, this enhances $\Delta_{m}$ and therefore modifies the wave functions of the ground state doublet, which gradually deviate from the pure $| \pm m\rangle$ spin projections (see Fig. D.6). This effect reflects itself in the decrease of the equilibrium longitudinal susceptibility $\chi_{z}$, by a factor close to 2 , which is observed experimentally and shown in Fig. D.9a. It also tends to weaken the dipolar bias $\psi$ by approximately same relative amount (remember that both $\chi_{z}$ and $\psi$ scale with $\left.\left\langle S_{z}^{2}\right\rangle\right)$. By integrating over the distribution function, one can then estimate the fraction $f_{\mathrm{Q}}$ of $\mathrm{Fe}_{8}$ molecules that, at any time, fulfill the condition $\xi<\Delta_{10}$. As expected, and as Fig. D.9b shows, $f_{Q}$ increases with $H_{y}$ and approaches $10 \%$ near 2 T. For these molecules, the magnetic ground doublet is characterized by fully delocalized wave functions. Step 1 then involves a delocalization of the bit states, e.g. from $|0\rangle$ to $|0\rangle \pm|1\rangle$ rather than a classical fluctuation between ' 0 ' and ' 1 ', whereas step 2 is a re-initialization of the wave function to a pure state, i.e. from $|0\rangle \pm|1\rangle$ to $|1\rangle$. 


\section{Appendix E: Uncertainty estimation}

The values of the susceptibility $\chi_{z}^{\prime}$ given in Fig.2 at each magnetic field result from averaging over $n=15$ samples. The uncertainty on the mean, $\sigma_{\chi}$, is calculated as its standard deviation assuming a normal distribution. The magnetization per molecule $M_{z}$ is a function of $\chi_{z}^{\prime}$ and the number of molecules $N$ and is therefore affected by an uncertainty $\sigma_{M}\left(H_{z}\right)$ given by the propagated uncertainties:

$$
\sigma_{M}\left(H_{z}\right)=\sqrt{\left(\frac{\partial M_{z}}{\partial \chi_{z}^{\prime}}\right)^{2} \sigma_{\chi}^{2}+\left(\frac{\partial M_{z}}{\partial N}\right)^{2} \sigma_{N}^{2}}
$$

Since the work $W_{2,4}$ is calculated as:

$$
W_{2,4}=\oint M_{z}\left(H_{z}\right) d H_{z}
$$

its upper $(+)$ and lower $(-)$ confidence bounds are given by:

$$
W_{2,4}^{ \pm}=\oint\left(M_{z}\left(H_{z}\right) \pm \sigma_{M}\left(H_{z}\right)\right) d H_{z},
$$

So that its associated standard deviation $\sigma_{2,4}$ is:

$$
\sigma_{2,4}=\left|W_{2,4}-W_{2,4}^{ \pm}\right| .
$$

The uncertainty, $\sigma_{1,3}$, on the work $W_{1,3}$ - obtained by integrating $M_{y}$-is calculated using an analogous procedure.

The total error affecting the work $W=W_{1,3}+W_{2,4}$ is then $\sigma=\sqrt{\sigma_{1,3}^{2}+\sigma_{2,4}^{2}}$.

\section{Appendix F: Details on the energy-time cost of a computation}

Here we report a more complete version of the chart in figure 4 of the main text where the quantities $W$ and $\tau$ determining the product $W \cdot \tau$ are plotted on the Cartesian plane (Fig. F.10). Along the diagonal line, at the top-right corner of the plane sit slow and lossy devices whereas the fast and efficient ones are on the bottom-left. The devices belonging to this corner of the plane are bounded from below by the quantum limit $W \cdot \tau_{\text {rel }}=\pi \hbar / 2=1.65 \cdot 10^{-27} \mathrm{erg} / \mathrm{bit} \cdot \mathrm{s}$.
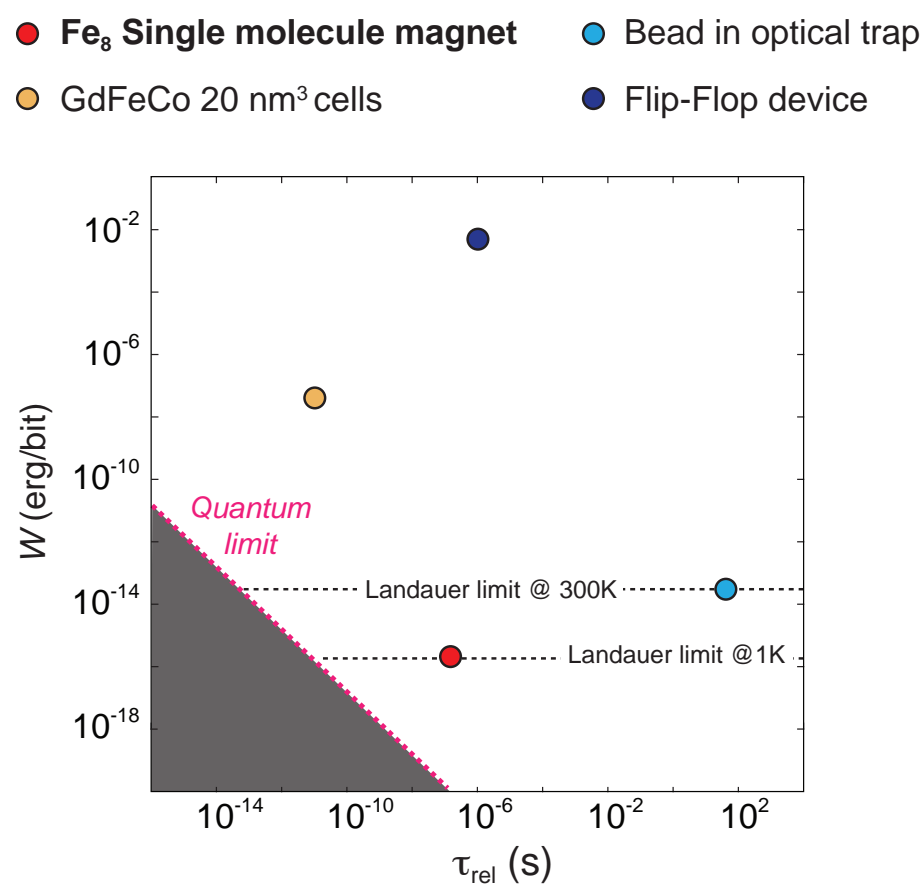

FIG. F.10. Relaxation time and efficiency comparison. (a) Chart comparing the energy-time cost of a storage operation performed with various systems. The $\mathrm{Fe}_{8}$ is the closest to the quantum limit. 